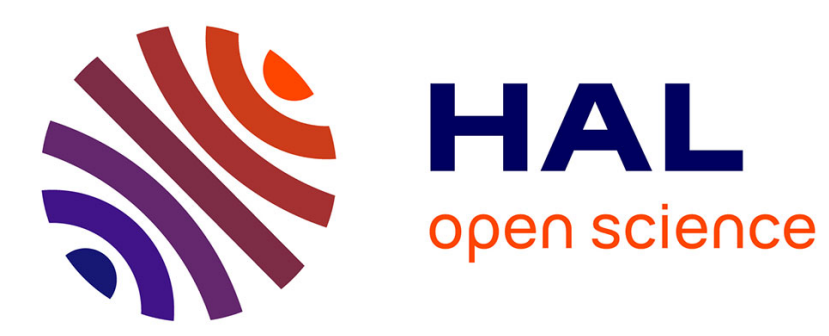

\title{
Zero mode counting in the presence of background fluxes
} Waldemar Schulgin

\section{To cite this version:}

Waldemar Schulgin. Zero mode counting in the presence of background fluxes. The Proceedings of the International Conference on Strings and Branes: The Present Paradigm for Gauge Interactions and Cosmology (Cargèse School on String Theory), 2006, France. hal-00250066

\section{HAL Id: hal-00250066 https://hal.science/hal-00250066}

Submitted on 8 Feb 2008

HAL is a multi-disciplinary open access archive for the deposit and dissemination of scientific research documents, whether they are published or not. The documents may come from teaching and research institutions in France or abroad, or from public or private research centers.
L'archive ouverte pluridisciplinaire HAL, est destinée au dépôt et à la diffusion de documents scientifiques de niveau recherche, publiés ou non, émanant des établissements d'enseignement et de recherche français ou étrangers, des laboratoires publics ou privés. 


\title{
Zero mode counting in the presence of background fluxes
}

\author{
Waldemar Schulgin*†ab,

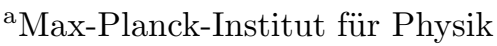 \\ Föhringer Ring 6, 80805 München, Germany \\ ${ }^{\mathrm{b}} \mathrm{LPT}$ ENS \\ 24 rue Lhomond, 75231 Paris Cedex 05, France
}

To obtain a superpotential contribution from a D3-instanton in type IIB string theory, two fermionic zero modes should be present on the worldvolume of a D3-brane wrapping a four-cycle. We discuss this criterium in the presence of fluxes for different configurations of $O$-planes and Euclidean $D 3$-branes.

\section{Introduction}

Compactifications of string theory to four dimensions usually produce massless scalar fields corresponding to the moduli of the compactified space. Observational absence of such fields forces us to find mechanisms which can make these fields massive. In the following we discuss the case of type IIB string theory. The complex structure moduli fields (also called shape moduli) usually obtain their mass by switching on vevs of the RR- and NSNS 3-forms in the ten dimensional theory [1]. The residual Kähler (or size) moduli fields could obtain their mass either from the terms in the higher $\alpha^{\prime}$ - expansion of the action or, as proposed by KKLT [2], by non-perturbative effects. These effects could be D3-instantons, i.e. Euclidean D3-branes wrapping four-cycles in the compact space, or gaugino condensates on the worldvolume of $D 7$-branes.

\section{Zero mode counting}

As shown in [3], if a divisor, wrapped by a $M 5$ brane in the dual $M$-theory picture (dual to type $I I B$ which one is considering), has holomorphic Euler characteristic

$$
\chi=h^{(0,0)}-h^{(0,1)}+h^{(0,2)}-h^{(0,3)}=1,
$$

the necessary two fermionic zero modes for the instanton contribution will be present. Note that for $h^{(0,2)}, h^{(0,3)} \neq 0$ this is only a necessary condition.

\footnotetext{
*schulgin@theorie.physik.uni-muenchen.de

†since 01.10.2007 schulgin@lpt.ens.fr
}

Orbifolds of type $Z_{N}$ and $Z_{N} \times Z_{M}$ were discussed with the aim to apply the KKLT procedure in [4]. Since there is no direct instanton calculation for the contribution of the $D 3$-instanton one should check at least the $\chi$-criterium. ${ }^{3}$ In a series of papers [6-10] it was shown how the zero mode counting and thus $\chi$ is changed in the presence of background fluxes. Additionally, it was shown in [11] that only the $(2,1)$-component of the $G_{3^{-}}$ flux may lift zero modes. The correspondence between zero modes of the Dirac operator on the worldvolume of the 4-cycles and Hodge numbers $h^{(0,0)}, h^{(0,1)}, h^{(0,2)}$ of these cycles becomes apparent by mapping the spinors to $(0, p)$-differential forms. Then fermionic zero modes of the Dirac operator correspond to the harmonic forms by this mapping. Locally we can write the world volume spinors on the D3-brane as

$$
\begin{aligned}
& \epsilon_{+}=\phi\left|\Omega>+\phi_{\bar{a}} \gamma^{\bar{a}}\right| \Omega>+\phi_{\overline{a b}} \gamma^{\overline{a b}} \mid \Omega>, \\
& \epsilon_{-}=\phi_{\bar{z}} \gamma^{\bar{z}}\left|\Omega>+\phi_{\overline{a z}} \gamma^{\overline{a z}} \Omega>+\phi_{\overline{a b z}} \gamma^{\overline{a b z}}\right| \Omega>,
\end{aligned}
$$

where $\epsilon_{+}$and $\epsilon_{-}$are states with positive and negative chirality with respect to the normal bundle $S O(2)$ of the $D 3$-brane inside the compact space. $a, b$ are the $D 3$-brane worldvolume directions and $z$ is the normal direction to the worldvolume. If one takes background fluxes, orientifold action and fixing of the $\kappa$-symmetry into account, some of the zero modes could eventuallybe lifted and the index

$$
\chi_{D 3}=\frac{1}{2}\left(N_{+}-N_{-}\right)
$$

\footnotetext{
${ }^{3}$ See however the recent attemps to calculate these contributions at the orbifold point [5].
} 
Table 1

Zero modes after fixing $\kappa$-symmetry and orientifold projection

\begin{tabular}{|c|c|c|c|c|c|}
\hline \multirow[b]{2}{*}{ chirality } & $\begin{array}{l}\text { O-plane } \\
\text { on top of D3 }\end{array}$ & \multicolumn{2}{|c|}{$\begin{array}{l}\text { O-plane } \\
\text { intersects D3 }\end{array}$} & \multicolumn{2}{|c|}{$\begin{array}{l}\text { O-plane } \\
\text { does not intersect D3 }\end{array}$} \\
\hline & $+\quad-$ & + & - & + & - \\
\hline$\overline{h^{(0,0)}}$ & $\phi$ & $\phi$ & & $\phi$ & $\phi \overline{a b z}$ \\
\hline$h^{(0,1)}$ & $\phi_{\overline{a z}}$ & {$\left[\phi_{\bar{a}}\right]$} & $\phi \overline{a z}$ & {$\left[\phi_{\bar{a}}\right]$} & $\phi \overline{a z}$ \\
\hline$h^{(0,2)}$ & {$\left[\phi_{\overline{a b}}\right]$} & & $\phi_{\bar{z}}$ & {$\left[\phi_{\overline{a b}}\right]$} & $\phi_{\bar{z}}$ \\
\hline \# of zero modes & $2-2 h_{(-)}^{(0,1)}+2\left[h_{(+)}^{(0,2)}\right]$ & $\begin{array}{l}2- \\
-2 h\end{array}$ & $\begin{array}{l}\text {,1) } \\
+2\left[h_{(+)}^{(0,1)}\right]\end{array}$ & $\begin{array}{l}2\left[h_{(}^{(()}\right. \\
-2 h_{(}^{(}\end{array}$ & $\begin{array}{l}+2\left[h_{(-)}^{(0,2)}\right] \\
-2\left[h_{(-)}^{(0,2)}\right]\end{array}$ \\
\hline
\end{tabular}

will change and $\chi_{D 3}$ is not anymore of purely geometrical nature. In the case of type $I I B$, Bergshoeff et al. [9] showed that only $h^{(0,1)}$ and $h^{(0,2)}$ of $N_{+}$can be lifted by fluxes. Thus, if the topology of the divisor has vanishing $h^{(0,1)}$, $h^{(0,2)}$, we can neglect the effect of the fluxes altogether and concentrate only on the action of the $O$-planes on the zero mode counting.

\section{Deformation of $\chi$ in the presence of fluxes}

Locally, there are always only three different configurations of the $O 7$-plane relative to the divisor in question. We summarize the results of the action of the projector equations (i.e. fixing of the $\kappa$-symmetry and orientifold-action) in all three cases in the table 1 .

In the horizontal line we give the zero modes associated to the Hodge numbers $h^{(0,0)}, h^{(0,1)}, h^{(0,2)}$. ' '+' and '-' denote the chirality with respect to the normal bundle of the D3-brane. In brackets we put the modes which are in general lifted in the presence of fluxes, and in the last line we give the number of zero modes which are left.

\section{Acknowledgements}

I would like to thank Dieter Lüst, Susanne Reffert, Emanuel Scheidegger and Stephan Stieberger for collaboration on the project [12]. Furthermore, I would like to thank Michael Haack and Daniel Krefl. It is also a pleasure to thank the organizers of the Cargese 2006 String School. This work was supported in part by the Agence Nationale pour la Recherche, France, contract 05BLAN-0079-01.

\section{REFERENCES}

1. S. B. Giddings, S. Kachru and J. Polchinski, Phys. Rev. D 66, 106006 (2002) [arXiv:hepth/0105097].

2. S. Kachru, R. Kallosh, A. Linde and S. P. Trivedi, Phys. Rev. D 68, 046005 (2003) [arXiv:hep-th/0301240].

3. E. Witten, Nucl. Phys. B 474, 343 (1996) [arXiv:hep-th/9604030].

4. D. Lüst, S. Reffert, W. Schulgin and S. Stieberger, [arXiv:hep-th/0506090].

5. R. Blumenhagen, M. Cvetic and T. Weigand, arXiv:hep-th/0609191.

6. R. Kallosh and D. Sorokin, JHEP 0505, 005 (2005) [arXiv:hep-th/0501081].

7. N. Saulina, Nucl. Phys. B 720, 203 (2005) [arXiv:hep-th/0503125].

8. R. Kallosh, A.K. Kashani-Poor and A. Tomasiello, JHEP 0506, 069 (2005) [arXiv:hep-th/0503138].

9. E. Bergshoeff, R. Kallosh, A.K. KashaniPoor, D. Sorokin and A. Tomasiello, JHEP 0510, 102 (2005) [arXiv:hep-th/0507069].

10. J. Park, [arXiv:hep-th/0507091].

11. D. Lüst, S. Reffert, W. Schulgin and P.K. Tripathy, [arXiv:hep-th/0509082].

12. D. Lüst, S. Reffert, E. Scheidegger, W. Schulgin and S. Stieberger, [arXiv:hepth/0609013]. 